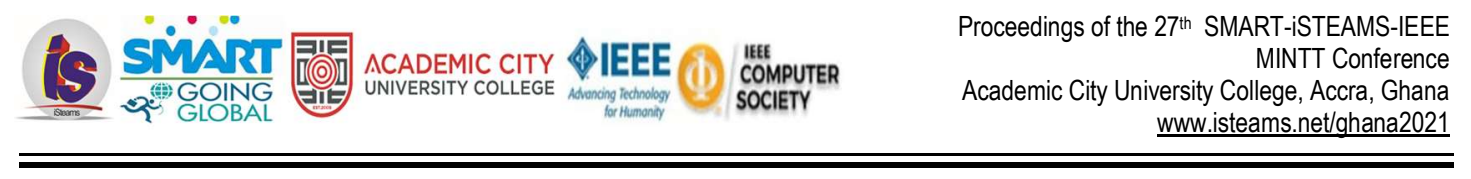

\title{
Quality of Service (Qos) Aware Power Saving Scheme in IEEE 802.16e Networks.
}

\author{
${ }^{1}$ Ajayi, E.A., ${ }^{2}$ dris, H, ${ }^{3}$ Wisdom, D.D. \& ${ }^{4}$ Arinze, U.C. \\ ${ }^{1}$ Faculty of Information Science \& Technology. Multimedia University, Maleka, Malaysia. \\ 2Department of Computer Science, Kaduna State University, Kaduna, Nigeria. \\ ${ }^{3}$ Department of Computer Science, Usmanu Danfodiyo University, Sokoto, Nigeria \\ 4Department of Computer Science, University of Nigeria, Nsukka, Nigeria. \\ E-mails: ebeseun@gmail.com; hajara.idris@kasu.edu.ng; danieldaudawisdom1@gmail.com, \\ arinzechuchukwugmail.com \\ Phones: +2347020396898; +2348036360999; +2347030743902; +2348066256878
}

\begin{abstract}
Abstract- Wireless telecommunication networks have become fundamental to our daily activities. Today, people have access to at least one type of wireless telecommunication network with Mobile Stations (MS) supporting multiple applications that consume more battery power; as well as a constant increase in multimedia applications that possess a critical challenge in efficient battery management. MSs are battery powered devices with limited lifetime. An efficient management of the battery will extend its lifetime before recharging exercise is conducted. World Wide Interoperability for Microwave Access (WiMAX), is design to Supports Higher Bandwidth with different traffic classes support for Power Management. Due to the mobility Characteristics introduced in WiMAX, power savings became an important problem; Since MS has limited superimpose life that requires recharging exercise when the battery life is depleted. Thus, Battery Lifetime Aware Power Saving Scheme (BLAPS) and an Adaptive Power Saving Scheme were proposed to extend the Battery Life, but the first scheme resulted in frequent transition to listening mode which led to waste of energy. While the former has higher energy consumption resulting to poor QoS that degrades the overall performance of MS. Hence, a Quality of Service (QoS) Aware Power Saving Scheme is proposed to improve the QoS. The scheme introduced a Modified minimum (Tmin) and Maximum (Tmax) sleep intervals in order to enhance the parameters of the variant schemes. In addition, the scheme also introduced a modified sleep window as well as QoS Aware algorithm that enable the adjustments of the sleep Parameters more appropriately in order to minimize frequent Transition to listening mode while improving efficiency. Finally, a state transition diagram was also developed. The proposed scheme was evaluated using MATLAB simulator; the results proved that the proposed QoS Aware Scheme has superior performance in terms of consumption rate and response delay respectively.
\end{abstract}

Keywords: Battery Life, Power Consumption, Response Delay. 


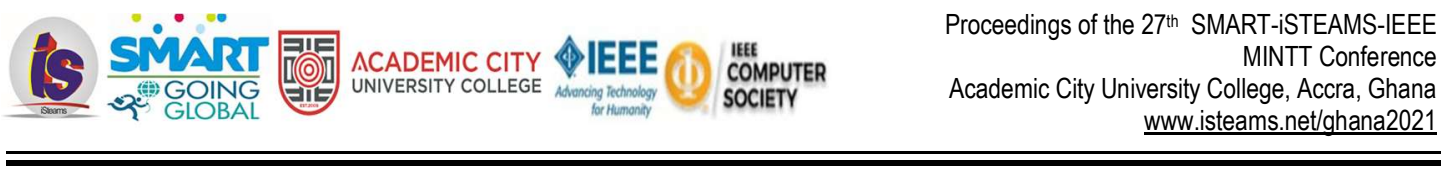

\section{INTRODUCTION}

For decades now, there had been advancement in worldwide interoperability for Microwave Access (WiMAX) also known as the IEEE 802.16, due to its low cost of deployment and higher bandwidth with different traffic classes' supports for efficient power savings. WiMAX is a Wireless Communication Technology providing numerous flexibilities in everyday lives. This advancement has been accelerated due to the proliferations of mobile devices such as smart phones, tablets, palmtops, PDAs, multimedia applications such as Voice over Internet Protocol (VolP), Internet Protocol Television (IPTV), interactive gaming and multimedia conferencing and the development of wireless network infrastructure.

These applications require high-speed broadband internet access. Therefore, broadband wireless access (BWA) technologies like WiMAX offer solution for high-speed internet access to users at a lower cost and also provide abundant access to Internet services. IEEE 802.16 standard popularly known as Worldwide Interoperability for Microwave Access (WIMAX) is one of the BWA technologies designed to provide such access for metropolitan area network. The standard was extended to IEEE $802.16 \mathrm{e}$ with the aim of providing mobility support for Mobile Stations (MS) (IEEE WG. 2004,2005).

These Mobility feature allows MS to move freely during services. Owing to the characteristics of this mobility in $802.16 \mathrm{e}$, a MS operates without continuous power outlet, hence MS operates depending on a rechargeable battery with limited capacity impose. The battery undergoes frequent energy drain due to the support it gives to multimedia applications. Therefore, power conservation is a critical challenge. Since, MS operates multiple multimedia applications with a limited superimpose lifetime known as energy Usually a MS waits for Mobile Traffic Indication Message (MOB-TRF-IND) from the base station (BS) in order to transmit or receive data.

The Energy of MS becomes wasted, especially when a MS waits for data and there is no data to receive; thus WiMAX uses a sleep window operation in order to conserve Power. The standard defines two modes for each MS: active mode and sleep mode. The active mode refers to a period of active service while the sleep mode is a period of inactivity. After each active mode, the MS sends a sleep request message to Base Station (BS) to initialize sleep mode. The BS sends an approval along with three sleep parameters: the initial sleep window (Tmin), the final sleep window (Tmax) and the listening window (L) to the MS to terminate from active data transmission and then proceeds to the sleep mode.

Several power saving mechanisms (PSM) have been proposed based on the sleep mode operation to extend battery life of the MS during traffic servicing (Vatsa et al. 2007). However, inefficient energy utilization becomes a critical challenge. Power Saving Classes (PSCs) are designed to address the above-mentioned challenges. Power Saving Class (PSC) of type I is used for Best Effort (BE) and other Non-Real Time (NRT) Traffics Such as Web and FTP files. PSC of type II is used for Real Time (RT) Traffics such as Unsolicited Grant Services (UGS), Voice over IP (VOIP).

PSC of type III is used for managing operations and multicast connections. Unlike PSC I and II, PSC III comprises of a single sleep window and is mainly used for multicast services (Figure 1). By activating this PSC, a single sleep window with defined length in WiMAX standard starts and subsequently the MS 
returns to normal mode operation. The summary of the IEEE QOS service classes is shown as follows in Table 1.

Table 1: Summary of IEEE QoS Service Classes

\begin{tabular}{|l|l|l|l|}
\hline Services & Description & Example & QoS \\
\hline $\begin{array}{l}\text { Real Time } \\
\text { Rariable }\end{array}$ & Real time data streaming for variable-sized data & $\begin{array}{l}\text { MPEG } \\
\text { Video }\end{array}$ & $\begin{array}{l}\text { High } \\
\text { Quality }\end{array}$ \\
\hline UGS & Real time data streaming for fixed-sized data & VOIP & $\begin{array}{l}\text { High } \\
\text { Quality }\end{array}$ \\
\hline $\begin{array}{l}\text { Extended } \\
\text { RT-VR }\end{array}$ & $\begin{array}{l}\text { IEEE 802.16e Added support real-time } \\
\text { applications with variable data rates }\end{array}$ & $\begin{array}{l}\text { VOIP With } \\
\text { Silence } \\
\text { Suppression }\end{array}$ & $\begin{array}{l}\text { High } \\
\text { Quality }\end{array}$ \\
\hline NRT-VR & $\begin{array}{l}\text { Delay Tolerant data streams for variable-sized } \\
\text { data }\end{array}$ & FTP & $\begin{array}{l}\text { High } \\
\text { Quality }\end{array}$ \\
\hline Best Effort & No minimum Service Level is required & $\begin{array}{l}\text { Web } \\
\text { Browsing } \\
\text { E-mail }\end{array}$ & $\begin{array}{l}\text { Low } \\
\text { Quality }\end{array}$ \\
\hline
\end{tabular}

Unsolicited grant Services (UGS), Non Real Time Variable Rate (NRT-VR), File Transfer Protocol (FTP) Voice over internet Protocol (VOIP).

PSC I:

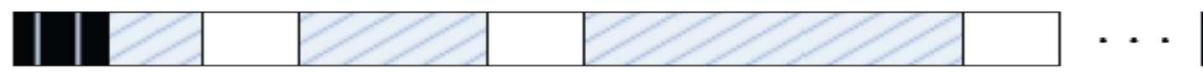

PSC II:
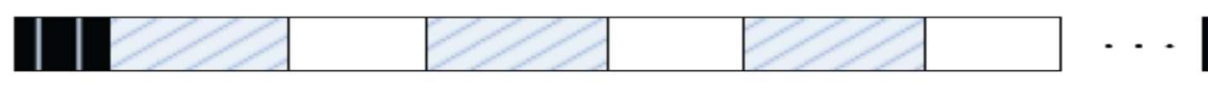

PSC III:

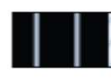

Figure 1: Types of Power Saving Classes I, II and III

These PSCs use three parameters to improve on power savings, namely, idle threshold, initial sleep window and final sleep window (Mehta et al. 2013, Zhu and Wang 2007). The idle threshold is the time interval in which the MS is in a waiting state, it has no messages to send or receive before moving to inactive state. The MS before moving to inactive state negotiates with it BS for approval in order to switch to a period of inactivity, if the request is granted by the BS the MS then switch to sleep mode otherwise repeats the Process (Figure 3). 

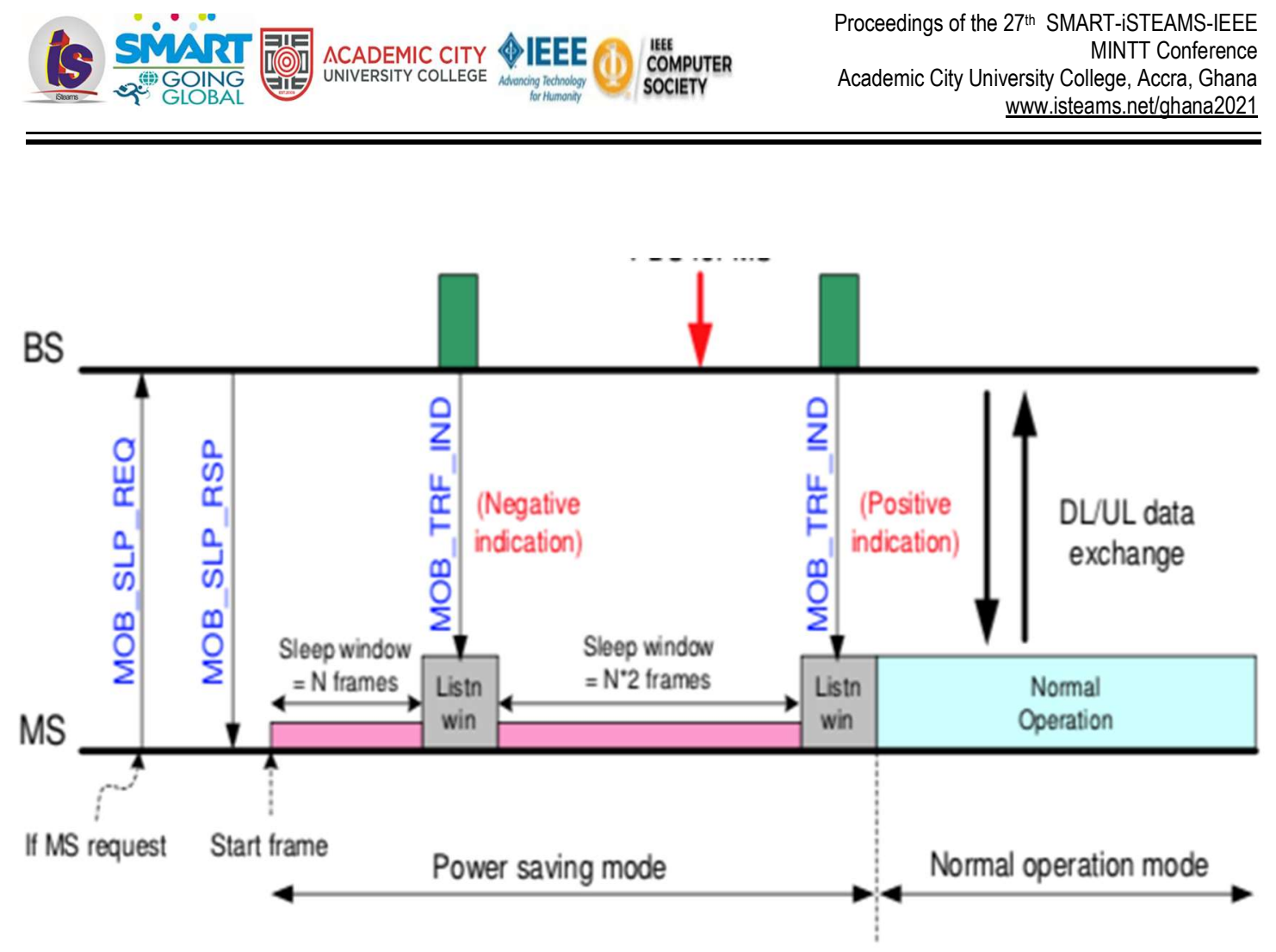

Figure 2: Sleep Mode Operation of PSC of Type I.

Figure 2 illustrates the schematic diagram of the IEEE 802.16e power-saving scheme, which includes the both operations of normal and the sleep mode. The MS begins by sending a request to the BS to enter the sleep mode; from idle state after a period of inactivity for a predefined idle time (T). The MS then negotiates with it serving BS by sending a mobile sleep request message (MOB SLP-REQ), when a positive mobile sleep response message (MOB SLP-RSP) is granted by the BS, the MS then switches to sleep mode. Within the sleep mode of PSC of type I, a specific number of sleep cycles are provided for the MS. And each sleep cycles consist of a sleep window and a listening window interleaved together with a fixed duration of time.

Based on the IEEE 802.16e standard for PSC of Type I, the MS is design to be in wake mode within the listening windows in order to monitor mobile traffic indication messages (MOB TRF-IND), which is a signal known as traffic indication message. At the sleep time (Period) the MS do not know anything until at the listening interval that is usually broadcasted from the BS. Where there are no DL data transmissions destined for the MS, a negative MOB TRF-IND value is sent to the MS. Upon receiving the negative MOB TRF-IND, the MS then continues staying in the sleep state. When repeated negative traffic indication messages are obtained by the MS, the length of its sleep window is then constantly doubled from the previous one until the maximum size of sleep window is reached where the MS maintains the sleep state otherwise end the process. 

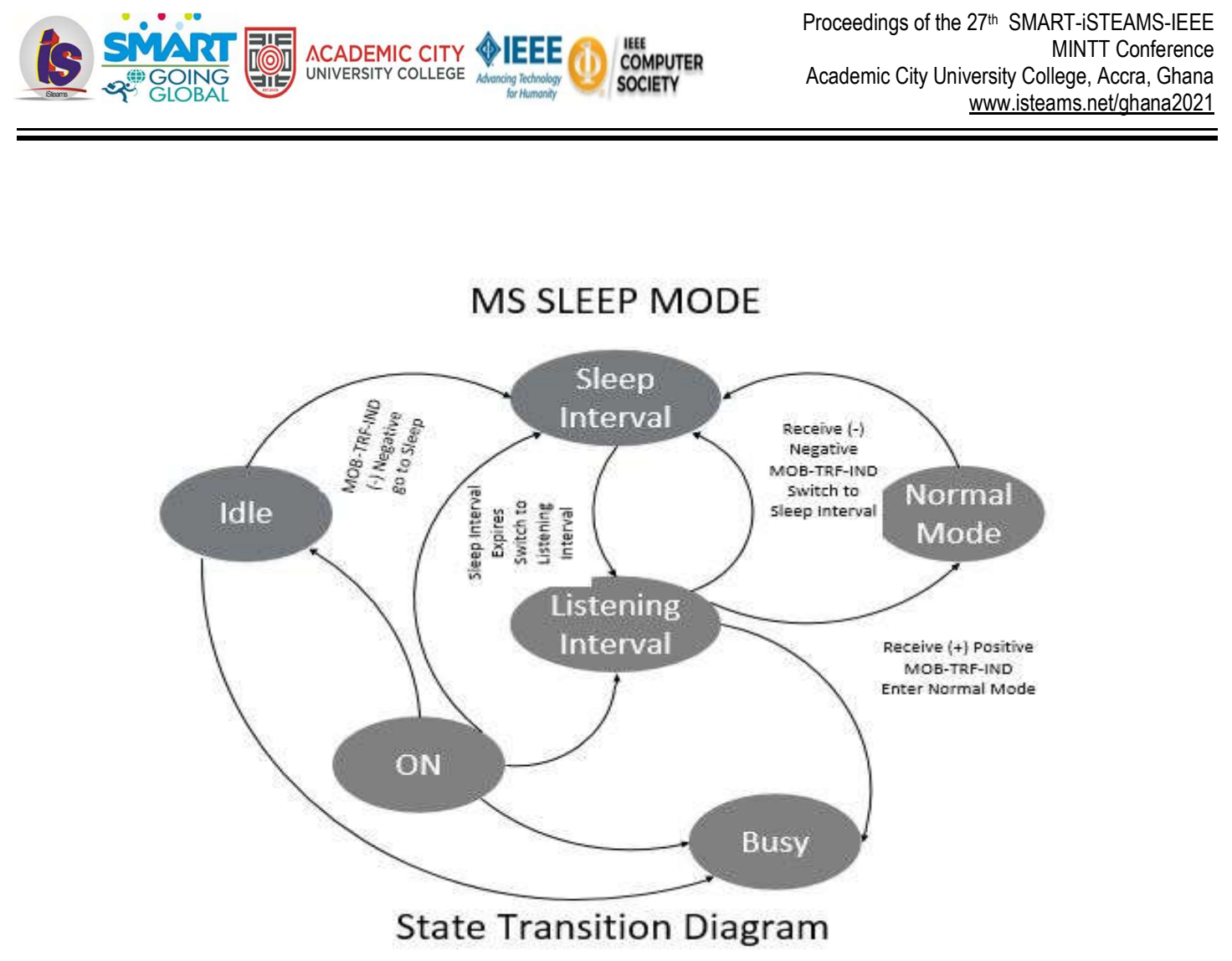

Figure 3: Proposed Procedure of Parameters Interaction.

The Figure 3: Illustrates the proposed schematic state transition diagram of the MS Sleep Mode. The MS begins from on (active) state and transits to listening state, from listening state the MS transits to busy state and or normal mode otherwise sleep state (interval). From sleep state the MS after a predefined time will switch on to anticipate data transmission, otherwise the MS is in idle state for a predefined time duration and receives a positive signal from the BS to either be in busy states so as to process data otherwise the signal given is negative, the MS then switch to sleep mode and maintain the period of its sleep time, as the sleep intervals expires a MOB-TRF-IND message is sent from the BS to MS to repeat the process as shown in Figure 2. Otherwise end the process.

The rest of this paper is organized as follows: Section II present Related Works, Section III Presents Proposed Algorithm, Section IV Presents Performance Evaluation and Section V Concludes this research paper.

\section{RELATED LITERATURES}

This section presents a review of related schemes on Power Saving Classes (PSC) that have been proposed in WiMAX Networks. The review focused on how the various propose power saving schemes used the main three sleep mode operating parameters to improve efficiency of MS. The way the proposed schemes control these sleep parameters were explored to further understand some of the inefficiencies and other open issues in power savings highlighted as follows: (Xiao, 2005) proposed a power scheme that considers MSs in sleep-mode at listening intervals but the MS is active. 
The scheme increased the sleep mode exponentially at negative MOB-TRF-IND Message traffic arrival from the BS. The scheme considerably reduced the frame response time and power consumption with an excessive listening operation, which results in waste of energy subsequently. Thus, an Enhanced Scheme was proposed in (Jufeng et al. 2016) to reduce the excessive listening operations of the existing power saving scheme. The Scheme used half of the previous sleep period in the next sleep- interval operation. When the initiate sleep interval is less than Tmin, then the initiate sleep interval is set to Tmin. The Base Station (BS) is notified of the initiate sleep interval request message by the MS. When the traffic is low the Inter-Service Data Unit (SDU) arrival interval is large enabling the Scheme to effectively decrease the number of listening intervals in one sleep-mode operation. The Scheme enhanced power management of MS.

However, it has higher delay due to the extended sleep intervals used. Hence, a Delay-Aware Auto Sleep Mode Operation was proposed in (Yang, 2007) to reduce the response delay as well as improve efficiency of MS. it dynamically adjust the sleep window according to traffic arrival and response requests, after serving all buffered packets; MS returns to sleep window which depends on the number of packets served. The scheme successfully minimized the response delay to a certain range. With a little increase in consumption.

Remaining Energy-Aware Power Management Scheme (REAPS) was proposed by (Min-Gon et al. 2008) to improve the performance of mobile devices. The scheme updates the sleep intervals dynamically taking into consideration the residual power and the inter-arrival of frames. The Tmax was updated using a smoothing technique with current inter-arrival time of a MAC SDU at each frame arrival and adjust Tmin by considering the power remaining and the Tmax. After the Scheme initialize parameters as namely: Tmin and Tmax and the current inter-arrival time of MAC SDU. It begins from normal mode operation and terminates when it receives positive request message to enter sleep-mode.

The scheme realises low-response delay if there is sufficient energy and prolongs the battery-life. However, it has little increase in energy consumption due to the constant Listening operations within the sleep window. Hence, an Efficient-Sleep-Window-Based Power Saving Scheme (ESPSS) in IEEE 802.16e Was Proposed in (Wisdom et al. 2019) to minimize power consumption and improve QoS, the scheme introduced an average based sleep window and a modified minimum and maximum sleep window to reduce the larger sleep intervals used and the delay of the existing Scheme. A discrete event simulator was used for the simulation, the simulation results of the proposed ESPSS Scheme proved that the ESPSS Scheme Successfully minimized the longer Sleep intervals and the response delay respectively while improving QoS. However, at higher traffics due to steady state of MS to transmit intending packets both schemes have similar performance.

A Dynamic Traffic Load-Aware scheme was proposed in (Jianbing et al. 2008) to improve the performance of MS. The scheme uses a dynamic approach by way of adjusting the idle check time of mobile devices which is the waiting $p$ intervals after the traffic arrival in wake-duration; before switching to the next sleepintervals. The algorithm is tuned dynamically after all buffered packet served according to the number of data-packets served and the preceding sleep intervals. The time is then set to zero when the last sleep window is larger than Tmin which makes Mobile devices go to sleep; but when the last sleep window is the same as Tmin it is set to a predefined value. 


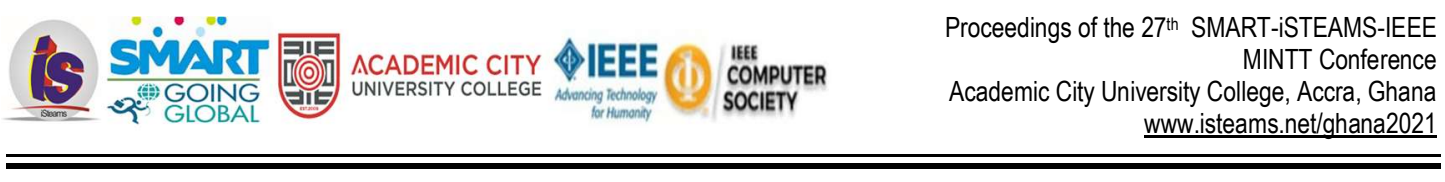

This makes mobile devices to wait for some time before transiting to sleep session. The algorithm increases power savings with a slight increase in algorithm complexity. A Scheme with periodic traffic indications was proposed in (Eunju et al. 2007) to reduce the longer response time of Mobile devices. The scheme used traffic indication messages to initiate transmission at every constant time. The TRFIND messages consist of a listening mode, awake mode and a sleep mode. During the listening state, a mobile device is synchronized with its serving BS as well as decides whether to switch to wake-mode or remain in a sleep-state. If there are data traffics in the buffer for the tagged MS, the BS sends a positive MOB-TRF-IND message and then transits to wake-mode. The BS sends data during the wake-state and terminate if no traffic arrival during a time-outfixed time of a constant length $\mathrm{T}$. If any data traffic arrives during inactive state, the mobile device transits to wake mode and transmits data packets, otherwise goes to sleep-mode from the wake-state without exchanging MOB-SLP-REQ/RSP messages.

The scheme reduced the average response delay because of its frequent switching from sleep/wake mode, with an increase in power-consumption. In (Saidu et al. 2017) a Hyper-Erlang Battery-Life Scheme (HBLES) was proposed to analytically tune the sleep parameters according to the residual battery power as well as traffic pattern to simultaneously reduce the consumption rate and the delay. The scheme used a Hyper-Erlang distribution to determine the actual traffic behaviour. The scheme improves the energy efficiency. But ignores uplink traffics. A Delay Aware Power Saving Scheme (DAPSS) based on load in traffic was proposed in (Wisdom et al. 2019) in order to reduce the longer sleep intervals of the existing Scheme. The scheme successfully minimized the longer sleep intervals of MS; thereby, reducing the average delay while improving power efficiency respectively.

However, the scheme ignores in-cooperating real time services with variant traffic characteristics, which may further affect the overall performance of the MS. Thus, an Enhanced power saving scheme based on load in traffic was proposed in (Wisdom et al. 2020) which is an extended version of DAPSS Based on load in Traffic, the Scheme enhanced the variant traffic characteristics of the existing DAPSS Scheme and successfully extends the battery life performance with a slight trade off increase in consumption rate of MS. Thus, Improving Efficiency with a Battery Life Time Aware Power Saving Scheme in IEEE 802.16e Networks was proposed in (Wisdom et al. 2020) to cooperates real time services by unifying PSC of both type I and II. The propose scheme analytically modified the sleep parameters based on traffic load and remaining battery power. It employed Hyper-Erlang distribution to determine the actual variant traffic characteristics and also uses an improved sleep mode control algorithm to resolve challenges of delay, buffer overrun and packet loss.

Our Simulation results showed that, the propose scheme has better performance compared to existing one in terms of average response delay with little cost of consumption rate. Hence, a Battery LifetimeAware Power Saving Scheme was proposed in (Chou et al. 2013) to improve mobile devices performance. The scheme dynamically adjusts the sleep parameters, according to the remaining battery power as well as traffic arrival. The scheme successfully extends the battery lifetime with an increase in consumption, more so the scheme frequently goes to listening mode if the traffic arrival is low thereby causing an average increase in the power consumption. In addition, an Adaptive Power saving Scheme was also proposed (Lin and Wang 2013) to extend the battery life of MS, the scheme successfully minimized the longer sleep time and improve performance of MS but resulted in average increase in energy consumption. 
This is due to inappropriate adjustments of the sleep parameters, which are the motivation for our research study. Hence, in this paper, a QoS Aware Power Saving scheme is proposed to address the above-mentioned challenges as well as improve QoS.

\section{PROPOSED QOS AWARE POWER SAVING SCHEME IN IEEE 802.16E NETWORKS}

A Quality of Service (QoS) Aware Power Saving Scheme for Mobile Broadband Wireless Access technologies (BWA) is proposed to minimize the frequent transition of MS to listening mode as well as higher rate of consumption of MS and improve QoS. The Proposed QoS Aware Power Saving Scheme is a Modification of the variant existing schemes namely: Adaptive Power Saving Scheme and (AdaptivePSS), and a Battery Lifetime Aware Power Saving Scheme (BLAPS). Previously, BLAPS was proposed to extend the battery life of MS. However, the BLAPS Scheme frequently goes to listening mode which results to waste of energy. Similarly, an Adaptive Power Saving Scheme was also proposed but the scheme subsequently results to higher energy consumption due to in-appropriate choice of the sleep parameters as well as the way of the adjustments of the parameters. The BLAPS scheme dynamically adjusts the three-sleep mode operating parameters namely: Iddle threshold $(\mathrm{Tt})$, Minimum sleep intervals (Tmin) and Maximum Sleep intervals to reduce the energy consumption. But the scheme extends the battery life of MS, at the expense of an increase in longer sleep window, which resulted to congestion, packet loss and performance degradation of MS.

More so, the increase in longer sleep intervals resulted to Poor QoS and an increase in both delay and consumption rate due to inappropriate (larger sleep intervals) choice of sleep windows. In addition, the increase in power consumption in the existing adaptive power saving scheme was due to the switching (cost) time taken for a mobile device (s) to revert (return) from sleep to active mode respectively. Finally, to address the problems highlighted above; a QoS Aware Power Saving Scheme in IEEE 802.16e Networks is proposed.First, the scheme introduced an efficient modified sleep window (Tj) given in Equation (1) to reduce the longer sleep intervals. Unlike the existing schemes where the minimum sleep intervals (Tmin) is fixed (Equation 1) of the existing schemes. In the proposed QoS Aware Power Saving Scheme, an average based maximum sleep window is employed to appropriately adjust the sleep parameters. Our proposed QoS Aware Power Saving Scheme dynamically tune the sleep parameters more appropriately based on traffic load arrival and delay requirements in order to minimize the unnecessary high consumption rate as well as improved QoS accordingly. Secondly, the Proposed QoS Aware Scheme has also been analytically derived as follows. In the

Proposed Scheme, the sleep window takes an average of the sleep time to appropriately admit traffics within their life time before expiration occurs (Tk) given as follows:

$$
T_{k}= \begin{cases}\left(1+\frac{K}{\lambda}\right) 2^{k-1} T_{\mathrm{m} \text { in }}, & \text { if } \quad\left(1+\frac{K}{\lambda}\right) 2^{K-1} T_{\mathrm{m} \text { in }}<T_{\mathrm{max}}, \lambda \neq 0 \\ \frac{T_{k-1}+T_{\mathrm{max}}}{4}, & \text { Otherw is e }\end{cases}
$$


Where $T_{k}$ is the kth sleep window

$T_{\mathrm{m} \mathrm{in}}$ is the minimum sleep intervals; $T_{\mathrm{max}}$ is the Final sleep intervals; $\mathrm{k}$ is a positive integer.

$T_{\mathrm{m} \text { in }}$ is determined by examining the inter arrival time of a downlink frame(s) in order to minimize the average response delay the downlink frames may had incurred in waiting for the MS to wake up.

Note that sleep window and sleep intervals are same in this paper, and that, sleep interval is interleaved with listening intervals (Figure 1). While a sleep cicle is the sum of sleep interval plus listening intervals.

Then, the idle threshold is adopted from [11][20]computed as follows:

First, the minimum idle threshold is computed as:

$$
P_{\text {id d le }}=\frac{P_{\text {waking }-u p}}{E_{\text {idd le State }}}
$$

Where Piddle is the power consumption during the idle state. Pwaking up is the power consume during the sleep waking session.

Second, the minimum idle threshold is also derived as follows:

$$
T-\min =\frac{P_{\text {waking- } u p}}{E_{\text {iddle State }}}
$$

The idle threshold ( $\mathrm{Tt}$ ) is adaptively updated based on the downlink traffic arrival pattern to predict the best duration for the next idle threshold. This best duration provides a suited idle time that considerably minimizes response delay and consumption of MS which is given as follows:

$$
\left.T_{t}=\min \left(\max \left(\lceil\lambda . d f t\rceil, T_{t_{-}} \min \right), \max \left(\left\lceil\left(\frac{\sigma_{\mathrm{n}}}{\mathrm{T}_{\text {average }}}\right) d f t\right)\right\rceil T_{t_{-} \mathrm{min}}\right)\right)
$$

Where Tt is the idle threshold, Tt-dft is the default idle threshold adopted from [19].

Next, the modified minimum sleep interval (Tmin) is obtained as follows:

First, the weighted average inter-arrival time (Taverage) in between the downlink frame from BS to the MS is obtained as follows: 
$T_{\text {average }}=(1-\alpha) T_{\text {min }}+\alpha T_{d}$

Tdis the time taken after which the DL frames arrive at the BS for MS since it went into sleep mode last, $\alpha$ is a positive integer. The weighted average variance $\left(\sigma_{n}\right)$ of the inter arrival time of the downlink frame, is also obtained as follows:

$$
\sigma_{n}=(1-\alpha) \sigma_{n-1}+\alpha\left|T_{\text {average }}-T_{d}\right|
$$

Finally, the modified minimum sleep interval (Tmin) is obtained as follows:

$$
T_{\mathrm{min}}=\max _{\operatorname{maverage}}\left(\left\lceil\sigma_{n}\right\rceil, 1\right)
$$

$\alpha$ andk are positive integers given as $0<\alpha<1$ and $0<k<1$ The modified Tmin is dynamically adjusted base on the traffic load arrival in order to transmit/process packets appropriately or just in time. The appropriate adjustment of the Tmin predicts the next actual arrival of the downlink frames which significantly minimize the average number of listening intervals in the sleep window.

Thus, the possible duration $(\operatorname{Pr})$ of a sequence of sleep cycles is dynamically calculated as follows:

$$
\operatorname{Pr}[k]=\sum_{k=1}^{\infty} \operatorname{Pr}(k=k)
$$

Finally, the modified final sleep interval (Tmax) is obtained as follows:

First, we assume that an incoming frame arrives at the MS during its idle state, and the probability ( $\mathrm{Pr}$ ) of frame arrival is given as follows:

$$
\begin{aligned}
& \operatorname{Pr}\left(n=T_{t}\right)=\operatorname{Pr}\left(e_{1}=\operatorname{tr} u e^{\prime}\right)=1-e^{-\lambda}\left(T_{k}+L\right) \\
& =\sum \sum_{k=1}^{\infty} \mathrm{P} \operatorname{r}(n=k) \sum_{k=1}\left(T_{k}+L\right)
\end{aligned}
$$

Second, we assumed that there is at least one frame arrival at the MS in the kth sleep window. Which implies that no packets in the 1st, 2nd, 3rd up to $(k-1)$ th sleep interval but there is at least one arriving frame in the kth sleep interval.

The $(\mathrm{Pr})$ probability of frame arrival in the kth sleep interval is obtained as follows:

$\operatorname{Pr}(n=k)=\prod_{i=1}^{k-1} \operatorname{Pr}\left(e_{i}=\right.$ false $) \operatorname{Pr}\left(e_{k}=\right.$ true $)=e^{-\lambda \sum_{i=1}^{k-1}\left(T_{k}+L\right)} 1-e^{-\lambda\left(T_{k}+L\right)}$ 
Let $\mathrm{M}$ satisfy the following:

$$
\left(1+\frac{K}{\lambda}\right) 2^{\mathrm{k}-1} \mathrm{~T}_{\mathrm{min},} \quad \text { if }\left(1+\frac{K}{\lambda}\right) 2^{\mathrm{k}-1} \mathrm{~T}_{\mathrm{min}<} \mathrm{T}_{\mathrm{max}} \text {; }
$$

$M$ is an integer value.

Third, the kth sleep window is obtained as follows:

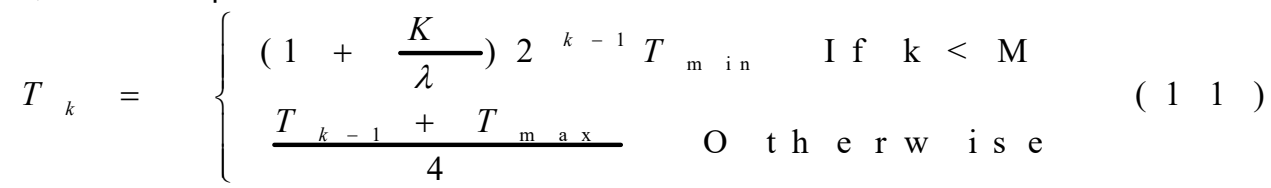

Assuming the packets resulting to network congestion from the sequence that results to poor QoS of sleep cycles arrives during the last cycle with uniform probability. The length of kth cycle is $\left(T_{k}+L\right)$ and the possible response delay of packets is obtained in Equation (11) as follows:

From Equation (1) above, we let M satisfy $\left(1+\frac{K}{\lambda}\right) 2^{M-1} \quad T_{\mathrm{m} \text { in }}=T_{\mathrm{m} \text { ax }}$

and $M$ is an integer. The kth sleep interval is given in Equation (11) above. And then we substitute Equation (11) into

Equation (10) and we have:

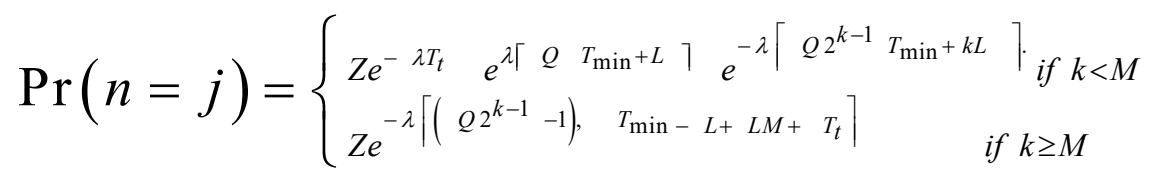

Where $Q=\left(1+\frac{K}{\lambda}\right)$ and $Z=\frac{T_{j-1}+T_{\max }}{4}$

Finally, Let $\mathrm{D}$ represents frame response delay and that the traffic arrival follows a Poisson distribution. The expected $(E)$ response delay is obtained as follows:

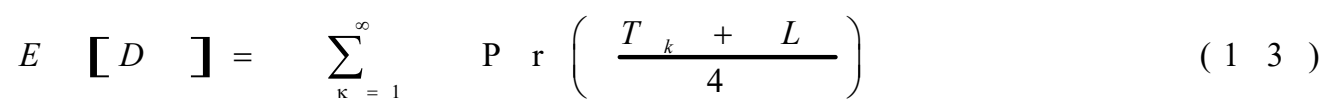

From Equation (13) and Equation (12) the expected response delay is expressed as follows:

$$
E[D]=\frac{L}{2}+\sum_{k=1}^{M} P_{r k} \frac{T_{k}}{2}+\frac{T_{k-1}+T_{\max }}{4(2)} \sum_{k=M}^{\infty} P_{r k} \quad\left(\begin{array}{ll}
1 & 4
\end{array}\right)
$$


From Equation (14) we have $\quad R=Q e^{-\lambda T_{t}} e^{\lambda\left\lceil Q \cdot T_{\min }+L\right\rceil \sum_{k=1}^{M-1} 2^{k-2}} e^{-\lambda\left\lceil Q .2^{k-1} T_{\min }+k L\right]} \cdot Z$

$\frac{T_{k-1+} T_{\max }}{8} \sum_{k=M}^{\infty} P_{r k}=\frac{T_{k-1}+T_{\max }}{8} e^{-\lambda\left(\left\lceil Q .2^{k-1}-1\right\rceil T_{\min -L+L M+T_{t}}\right)}$

Unlike the existing schemes that makes use of larger sleep windows and the full length of the Tmax sleep intervals Figure 1 and 2, which subsequently results in a longer sleep period (time). The proposed QoS Aware Scheme introduced an efficient sleep window that takes the average Tmax values in order to improve QoS.

More so, when the sleep intervals subsequently approaches final stage called the Tmax, the sleep intervals is increased incrementally with an exponential increase as an average of the kth sleep window (Equation 1 and 11)

To sufficiently conserve energy, we reduce the response delay the downlink frame may had incurred subsequently while appropriately or just in time processing/transmitting packets within their life time (Figure 2).

$\frac{T_{k-1}+T_{\mathrm{max}}}{8} \sum_{k=M}^{\infty} P_{k}=\frac{T_{k-1}+T_{\mathrm{max}}}{8} e^{-\lambda\left\lceil\left(Q .2^{k-1}-1\right) T_{\min -} L+L M+T_{t}\right\rceil}$

The sum of the average response delay of the Propose QoS Aware scheme is also expressed as follows:
$2 E$
$\left[\begin{array}{ll}D & ]= \\ \sum_{k}=1 & \infty\end{array}\right.$
$P_{k}\left(T_{k}+L\right)$
$\left(\begin{array}{lll}1 & 8\end{array}\right)$

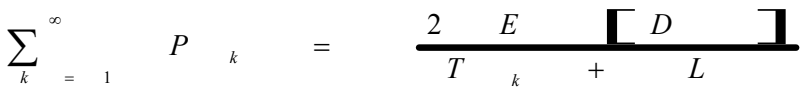
$\left(\begin{array}{lll}1 & 9 & \end{array}\right)$

Substituting 14 and 15 into 13 , we have

$$
T_{\max }=\frac{2 E[D]-L-2 R}{e^{-\lambda\left\lceil Q \cdot 2^{k-1}-1\right\rceil T_{\min }-L+L M+T_{t}}}-\frac{T_{k-1}}{8}
$$


Hence, the maximum sleep window is obtained as follows:

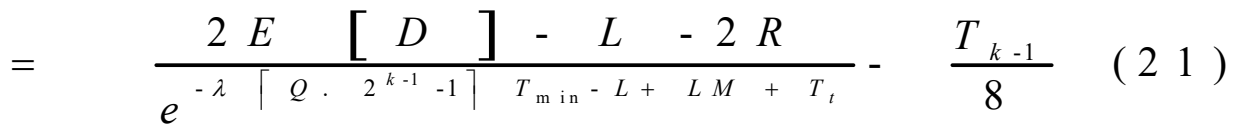

\subsection{Sleep Mode Operational Procedure}

When a MS is in sleep mode (Figure 1 and 2). MS Transits from sleep wake mode (Window). The MS waits in the sleep mode for a set period of time before switching to normal mode operations' MS sleeps for a set interval period of time and subsequently wakes up after the sleep time expires and then goes to the listening interval. During the listening interval, when a positive (+) MOB-TRF-IND message is received, the MS switches to normal mode. Otherwise, the MS goes to next sleep cycle and repeat it process [21].

Note that a sleep interval plus (+) a listening interval makes one complete sleep cycle, since sleep intervals are interleaved with a listening interval. MS takes Tmax as final sleep intervals and the length of the subsequent sleep intervals doubles the previous sleep intervals.

The Pseudo Codes of the Proposed QoS Aware Scheme That describes the step by step operations of MS in different states are given as follows in algorithm 1.

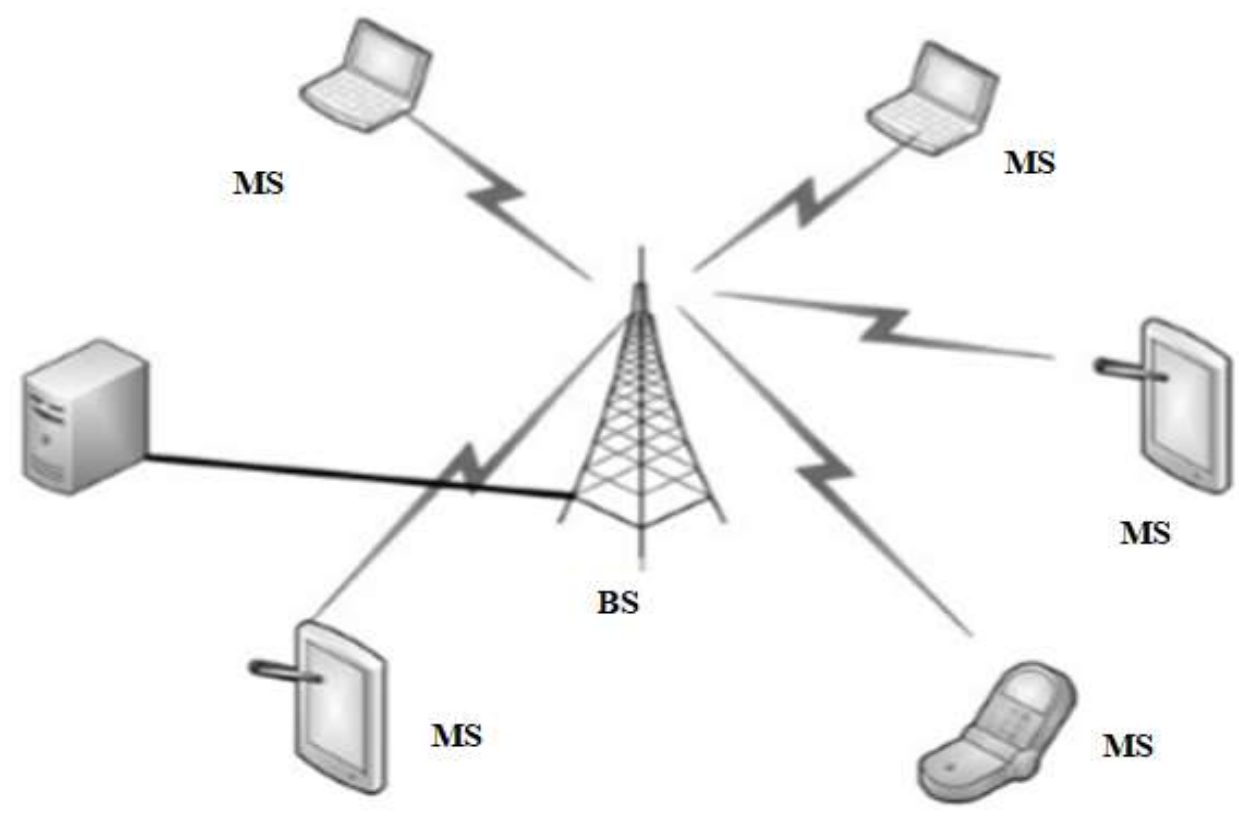

Figure 4. Simulation Topology. 
The above figure 4 illustrates simulation topology of a single Based Station (BS) consisting of five Mobile Stations sorounding it with a single server that stores, retrieve and sends data packets across the network.

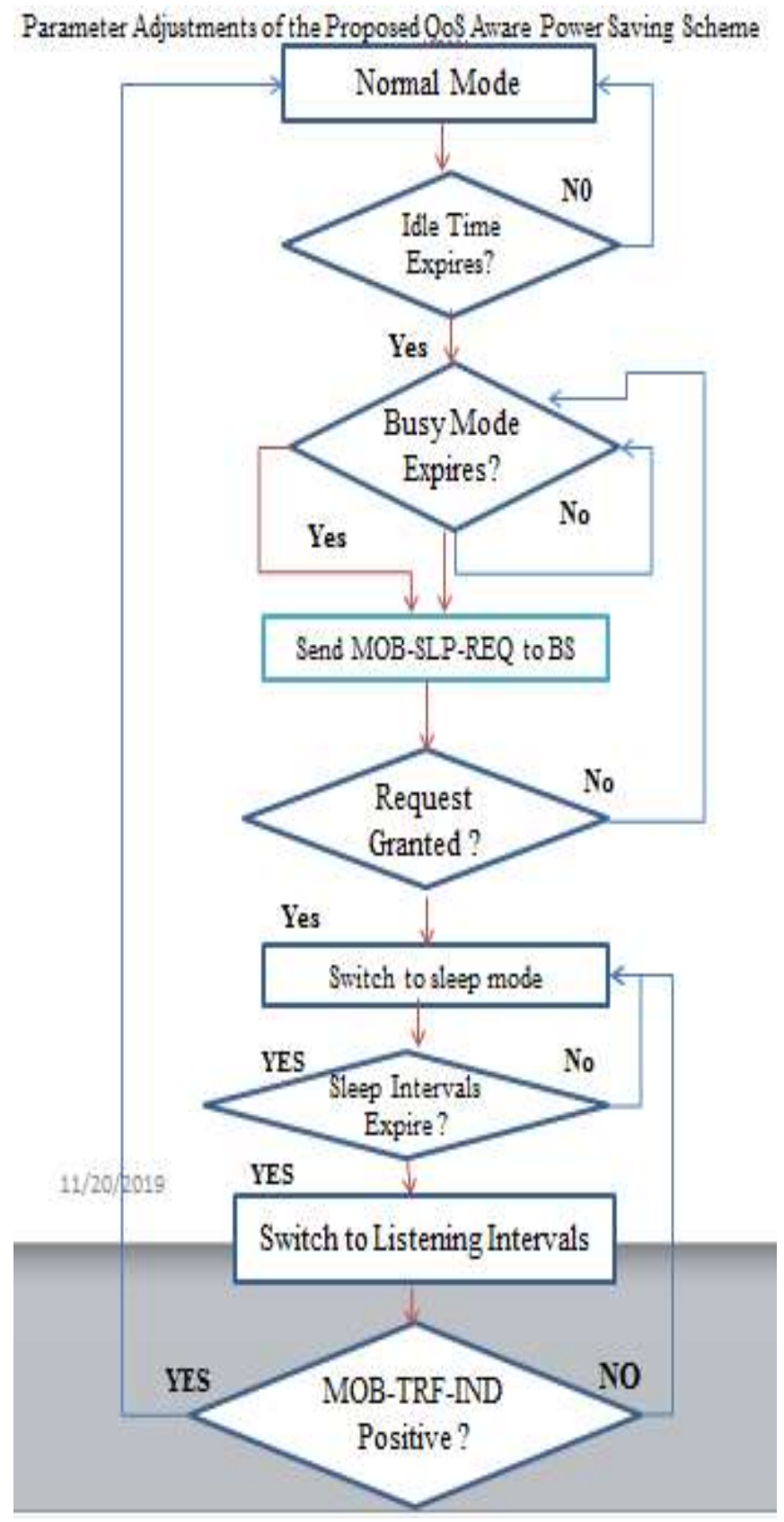

Figure 5. the Proposed Procedure of Parameters adjustments of the proposed QoS Aware Power Saving Scheme 
Figure 5 illustrates the Proposed Procedure of Parameters adjustments of the proposed QoS Aware Power Saving Scheme. The MS Begins from a normal mode procedure and check if the idle time is expired, if no it transits to normal mode operation and repeats the process, if yes the MS transits to busy mode. And checks if the busy mode is expired if no it will maintain the same and process traffics, if yes the Ms Then sends MOB-SLP-REQ Message to the BS. If request is not granted that implies the MS is still processing traffics, else is yes the MS then transits to sleep mode. From sleep mode if the sleep interval is not expired the MS will maintain it dedicated sleep states.

If the sleep mode expires then MS transits to listening interval, at the listening intervals MS anticipates Traffic indication from the BS. If the traffic indication is not positive MS reverts to sleep mode. Otherwise, Traffic indication is positive and the MS now transits to normal mode operation and continue the loop else end the process. 
Set Parameters: Input $\mathrm{k}=1$, Tmin_ $\lambda=\mathbf{k}$, Tmax $=1024$

Switch (States of MS)

Case 1: MS in Normal Mode?

Sends MOB-SLP-REQ Messages to BS

If (MS receives (+)positive MOB-SLP-REQ) \{

Ms Goes to Sleep Mode

\} Else $\{M O B-S L P-R E Q$ is not granted, $M S==$ Active

\} \#End of else

\} End of Case 1

Case 2: MS in Sleep Mode \{

If (Sleep Time Expires)

MS switch to Listening Interval ();

If (MS receives (+)positive MOB-TRF-IND Message)

\}Else\{

MS Transits to active state and Process Traffics

MS in idle Mode anticipating data packets + MOB-TRF-IND Messages

If (Idle time expires and no traffics arrival \{

MS Switch to Sleep Mode and remain in Sleep Mode until There is + MOB-TRF-IND

\}

\} \#End of Case 2

\} \#End of Switch Case

MS Checks (MOB-TRF-IND)\{ \#Recieves the MOB-TRF-IND Messages

If (Traffic Indication Bit $==1)\{$ \#it implies positive Traffic Indication

Call update ();

\} \#End of if

Else \{ Call Sleep (); \# implies Sleep Mode Operation in WiMAX

¿\#End Of Else

;\#End of MS check ()

Update () \# \#updates the setting parameters based on the Traffic Load

Call Load (MOB-SLP-RSP);

Calculate $T_{\min }=2{ }^{k} T_{\min } \lambda$

if $\left(T_{\min }<T_{\max }\right) k=k+1$ and repeat step 1

Else \{

calculate $T_{\text {max }}=2^{k} T_{\text {min }}-\lambda$

if $\left(\right.$ Power $\left(T_{\min } T_{\max , \lambda}\right)<\min$ PowerConsumption\& $\left(T_{\min } T_{\max }\right)<\min$ response Delay $)\left\{\quad T_{\min \_} \lambda=T_{\min } T_{\max } \lambda=T_{\max }\right.$

min_power Consumption $=\operatorname{Power}\left(T_{\min ,}, T_{\max , \lambda}\right)$

$\min \_$responseDelay $=\operatorname{Delay}\left(T_{\min ,} T_{\max , \lambda}\right)$

\}Else \{

Input $k$

do step 1

\} End of Else

\} \#End of Update ()

Load (MOB-SLP-RSP) \{ \#Load the parameter Settings From MOB-SLP-RSP

\#Tmin :=Minimum Sleep Interval, Tmax :=Final Sleep Interval and $\lambda=$ the arrival rate

\} \#End Load () 


\section{PERFORMANCE EVALUATION}

This section presents the performance evaluation of the propose QoS Aware Power Saving Scheme against that of the Existing Schemes. The evaluation is based on the average power savings and response delay respectively. The simulation topology consists of a base station (BS) with MS connected around it (Figure 4). In this study, MATLAB simulator was used for the performance evaluation of the Proposed QoS Aware Power Saving Scheme against the existing Adaptive power Saving Scheme and that of the Battery Lifetime Aware Power Saving Scheme. Results obtained at the simulation time are presented as follows:

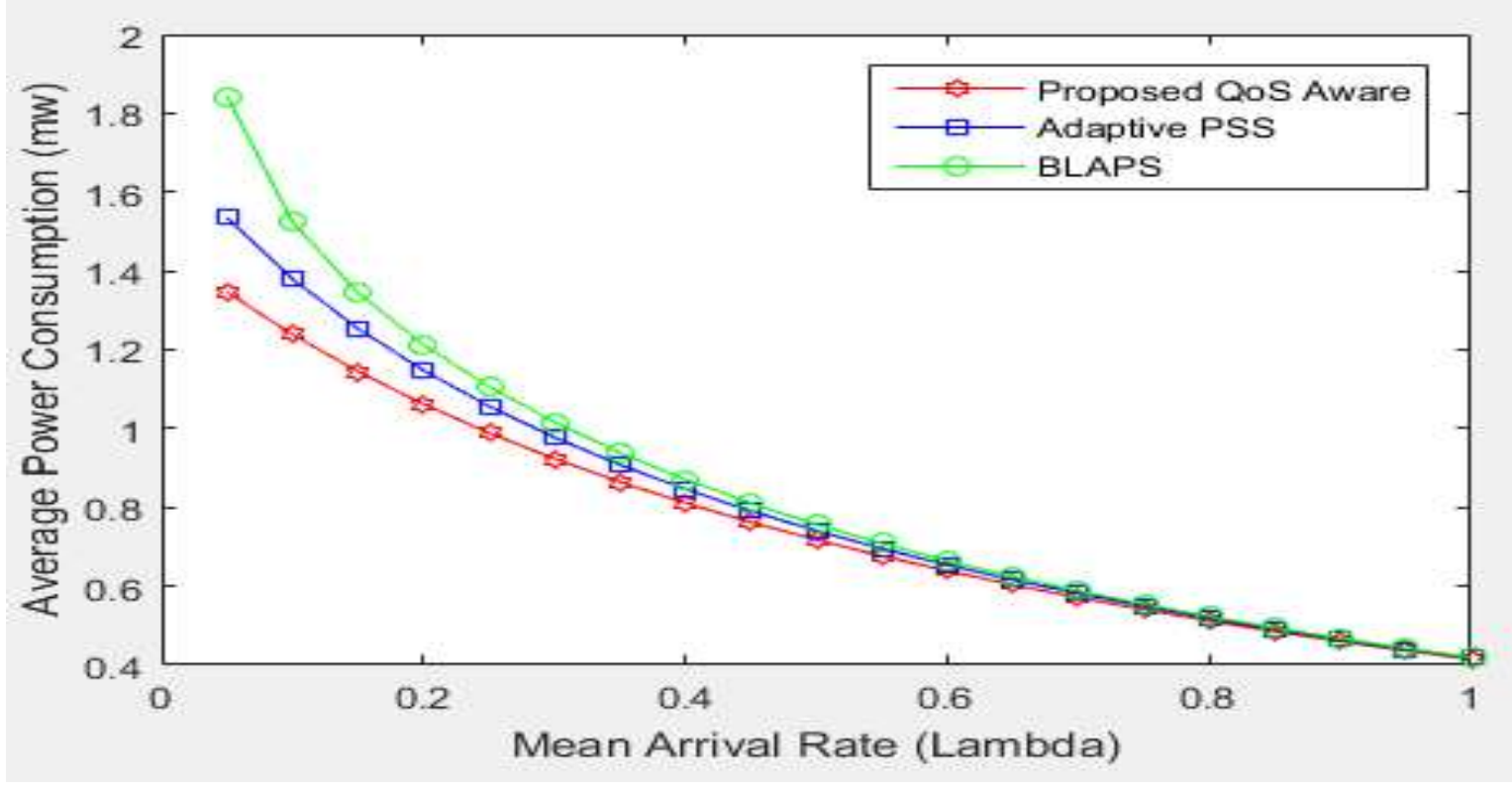

Figure 6: Average power consumption VS Mean arrival rates.

Figure 6: Illustrates average power consumption VS Mean arrival rates. We observed that, from 0 to 0.7 the Proposed QoS Aware Scheme has lower Consumption rate with a superior performance as compared to the existing BLAPS and Adaptive Power Saving Scheme (PSS). However, at a higher traffic arrival rate due to heavy traffic. Since at heavy traffic frequent switching is minimized because MS has to be in active state processing traffics and when the Mobile device (MS) Battery Life is becoming depleted the both schemes have similar performance. 


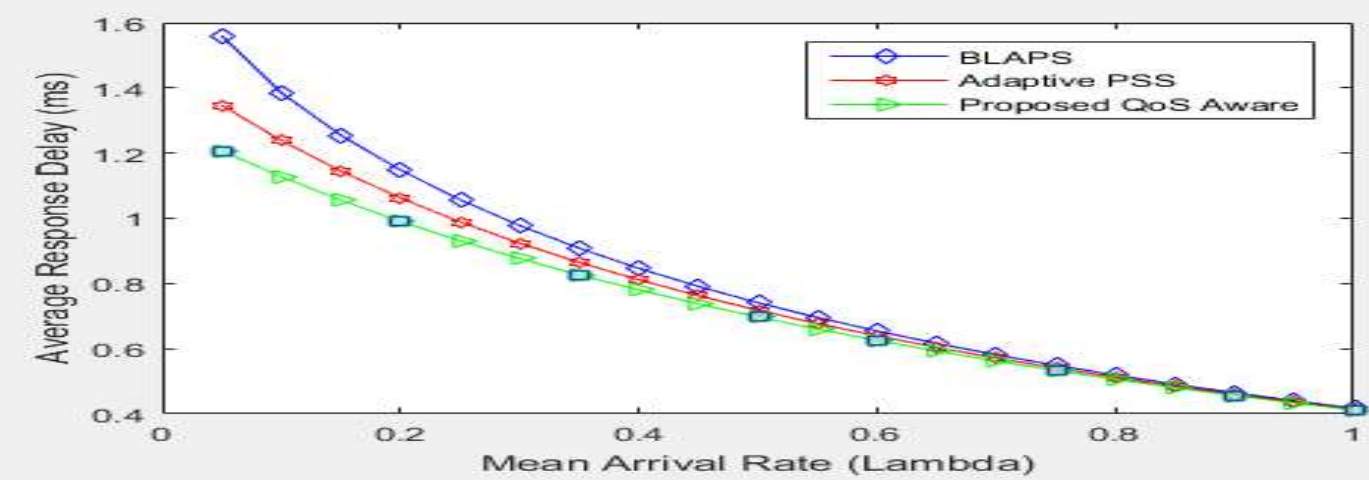

Figure 7: Average Response Delay VS Mean arrival rates

Figure 7: Illustrates average Response Delay VS Mean arrival rates. We observed that, from 0 to 0.6 the proposed QoS Aware Scheme have significantly minimized the longer sleep interval. Hence, minimizing the response delay due to the Modified Tmin and Tmax as well as the introduction of an efficient sleep window. However, when there are higher traffic arrivals at the threshold level, especially when the MS Battery life is becoming depleted both schemes have similar performance. Thus, the proposed QoS Aware Scheme and the existing schemes converge towards similar point respectively.

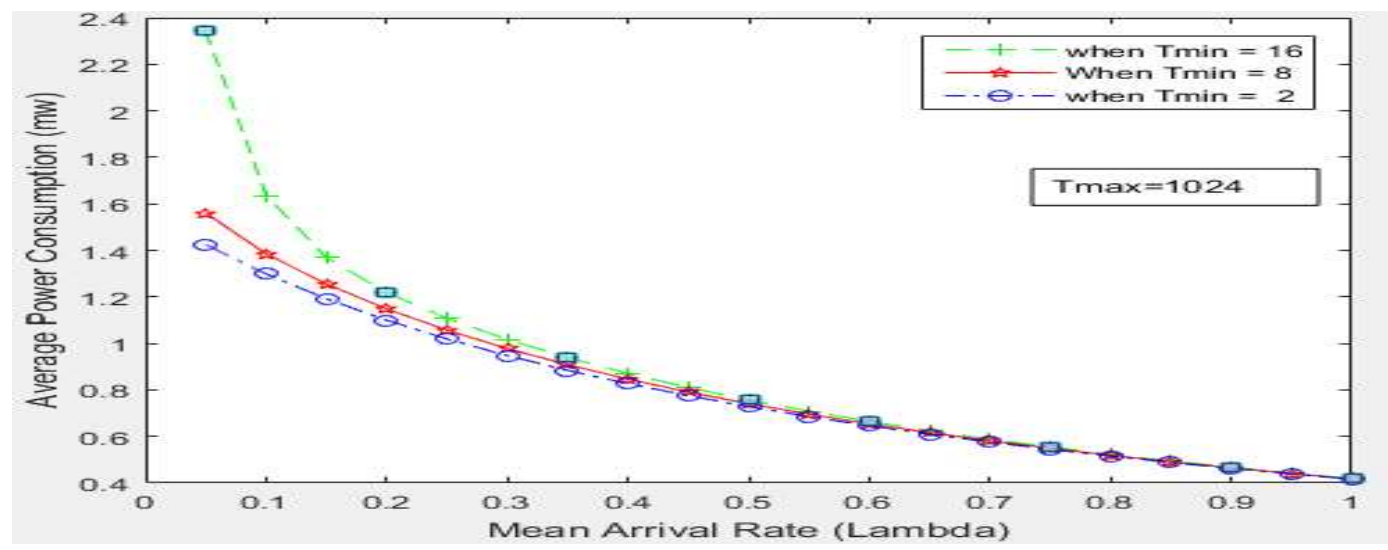

Figure 8: Average power consumption vs Mean arrival rate.

Figure 8 illustrates average power consumption vs Mean arrival rate. When Tmin is equal to 2, 8, and 16 and Tmax is 1024, we observed at the simulation time that when Tmin is equal to 16 higher consumption rate is experienced which results to resource wastage. However, datapackets are successfully transmitted with less issues of congesion/packet loss in the case of higher traffic arrival rate as comapared to when Tmin is 8 or 2 respectively. While when Tmax is equal to 1024 and Tmin is 2 lesser energy is consumed with a slight response delay due to frequent switching to sleep/wake Mode, however as the MS battery life becomes deplited it is observed that both scheme tend towards same point. 


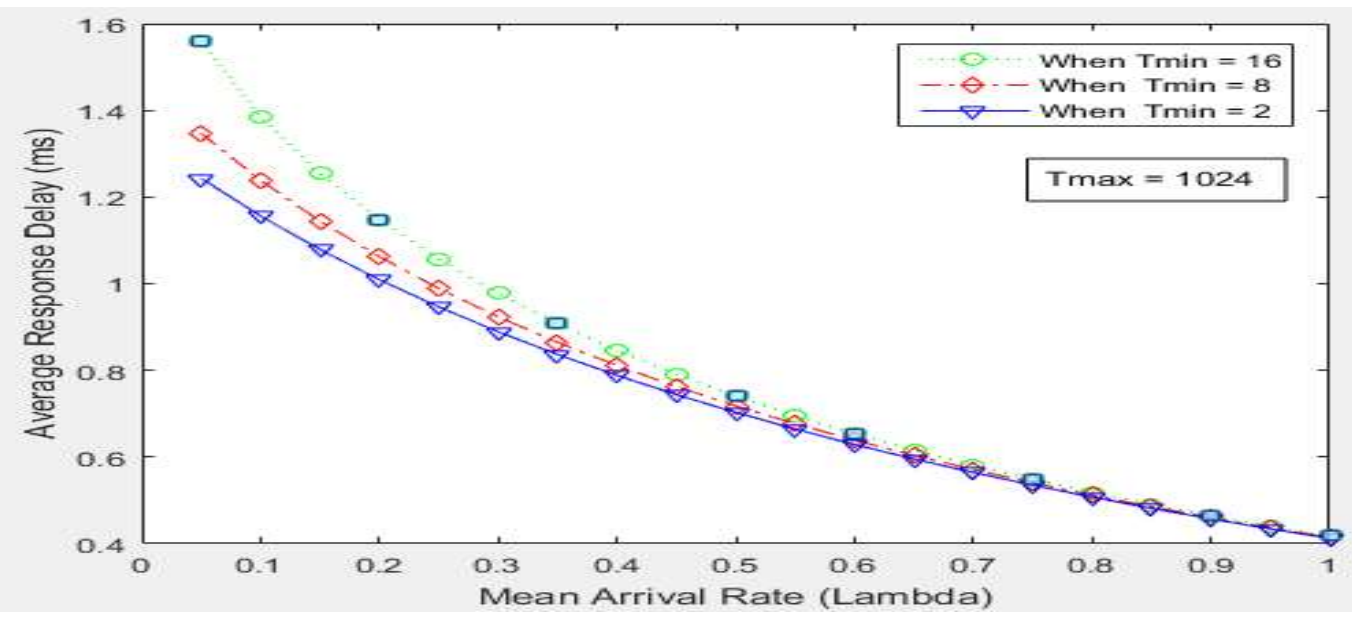

Figure 9: Average response delay vs Mean arrival rate, when Tmin is 2, 8, and 16 and Tmax is 1024.

Figure 9 illustrates average response delay vs Mean arrival rate, when Tmin is 2, 8 , and 16 and Tmax is 1024. from the bigining it is observed that when Tmin uses larger values such as 16 higher response delay is experienced which may likely results to cases of congestion or packet loss; while sufficient energy is saved comapared to when Tmin is 8 or 2 respectively. While when Tmax 1024 and Tmin is 2 less delay is encured, but with frequent switching from sleep wake mode. And since, after the MS enters sleep mode, the MS must wait for the next listening window to receive data, which usually increases the average response delay.

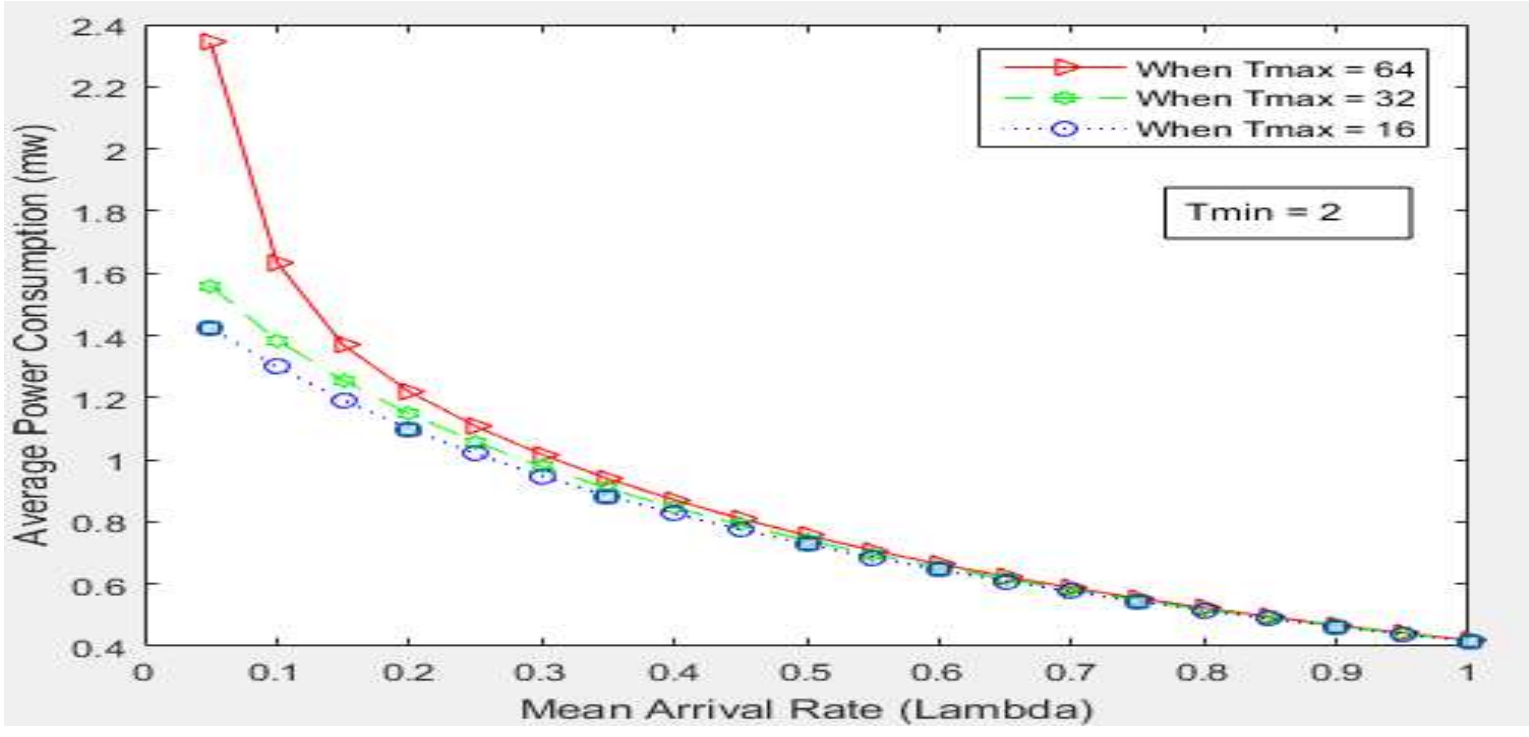

Figure 10: Average power consumption vs Mean arrival rate. When Tmax is equal to 16,32 , and 64 and Tmin is 2 
Figure 10 illustrates average power consumption vs Mean arrival rate. When Tmax is equal to 16,32 , and 64 and Tmin is 2, from the bigining at simulation time it was observed that when Tmax is equal to 64 higher consumption rate is experienced since MS has to be active at this time wether or not there are traffic arrival which results to resource wastage. However, datapackets are successfully transmitted sufficiently with less likely cases of congesion or packet loss in the case of higher traffic arrival rate as comapared to when Tmax is 32 or 16 respectively. While when Tmin is equal to 2 and Tmax is 16 lesser energy is consumed.

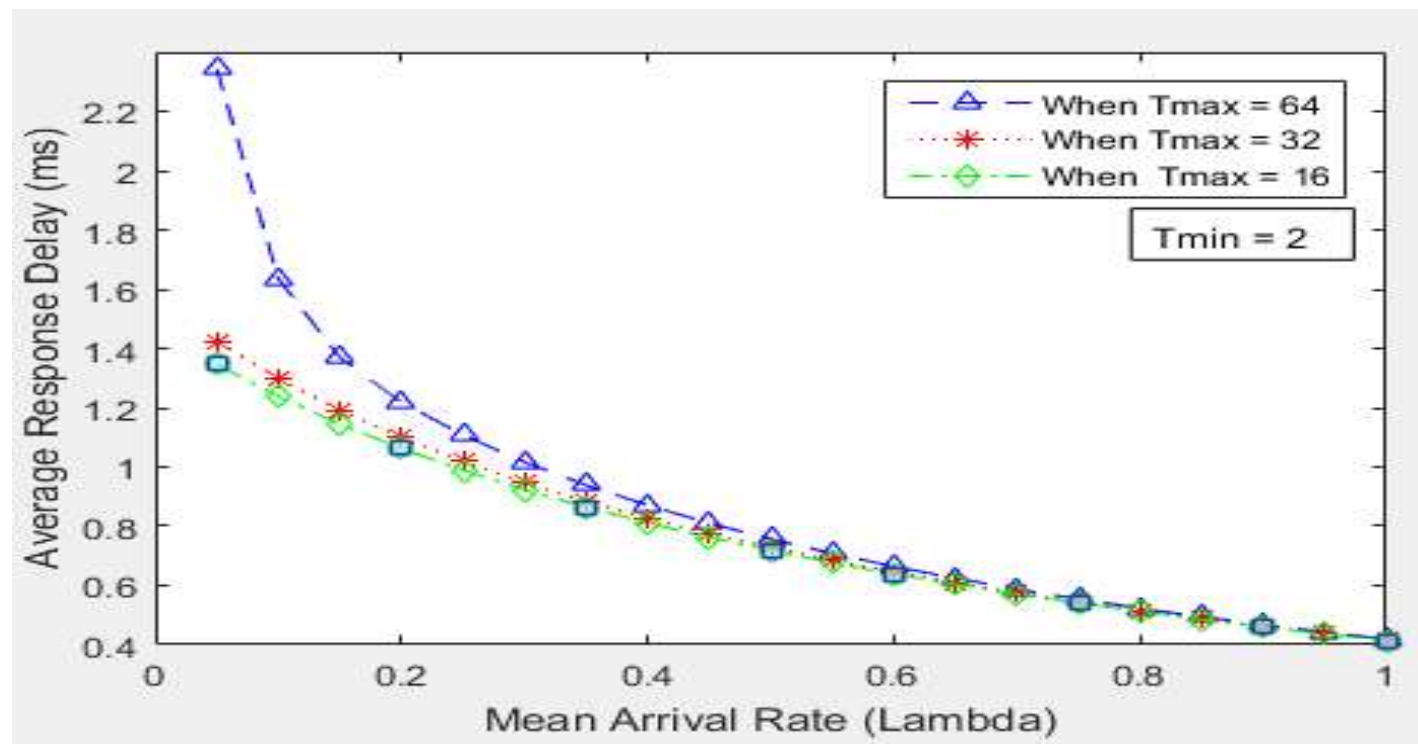

Figure 11: this illustrates the average response delay vs Mean arrival rate, when Tmax is equal to 16,32 , and 64 , while Tmin is equal to 2 .

Figure 11 illustrates the average response delay vs Mean arrival rate, when Tmax is equal to 16, 32, and 64, while Tmin is equal to 2 . At the simulation time, from the bigining it is observed that when Tmax is equal to 64 higher response delay is incured which may likely results to cases of congestion or packet loss; while sufficient energy is saved comapared to when Tmax is 32 or 16 respectively. While when Tmin is equal to 2 and Tmax is 16 lesser response delay is incured. However, frequent switching from sleep wake mode is experienced, and after the MS enters sleep mode, the MS must wait for the next listening window to receive data, which increases the average response delay.

\section{CONCLUSION}

A Quality of Services (QoS) Aware Power Saving Scheme for Mobile Broadband WiMAX Network is proposed; the scheme introduced a Modified minimum and maximum sleep intervals (Tmin and Tmax) as well as a modified Sleep Window. More so, the proposed scheme employed a modified QoS Aware Algorithm to improve efficiency of MS. The scheme dynamically adjusts the three sleep operating parameters namely Idle threshold, Tmin and Tmax just in time and significantly minimize the frequent 


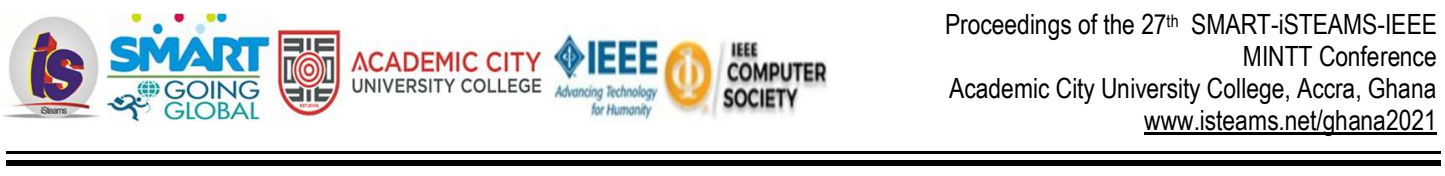

transition to listening mode of MS by way of selecting appropriate sleep parameters while minimized consumption rate as well as improved the QoS accordingly.

The Proposed scheme tunes the sleep parameters based on traffic load arrival and aptly choose the best listening/sleep time in order to save energy and improved QoS accordingly. Finally, the proposed scheme, analytical and simulation results are both presented, that determine the actual best duration of Sleep/listening mode. The proposed scheme also employed an efficient sleep window in order to admit traffics within their life time before expiration. The scheme was evaluated using a discrete event simulator (MATLAB), the simulation results depicted in Figures proved that the proposed Scheme perform better compared to the existing schemes both in terms of consumption rates and response delay.

\section{REFERENCES}

1. IEEE 802.16 WG, Standard for Local and Metropolitan Area Networks Part 16: Air Interface for Fixed Broadband Wireless Access Systems, IEEE 802.16 working Group and others, IEEE Std, 802.16, 2004.

2. IEEE 802.16e WG, IEEE Standard for Information Technology Telecommunications and Information Exchange between Systems-LAN/MAN Specific requirements, Part 16: Air Interface for Fixed and Mobile Broadband Wireless Access Systems, IEEE Std802.16e, 2005.

3. Vatsa, O. J., Raj, M., Ritesh Kumar, K., Panigrahy, D., and Das,D. (2007). Adaptive power saving algorithm for mobile subscriber station in 802.16e, In the 2nd IEEE International conference on communication systems software and middleware, 2007 (COMSWARE 2007), pp. 1-7.

4. Mehta, S. Seth, N. Sharma, N. Snigdha. (2013). A Novel Approach for Minimizing the Delay and Load in Wireless Network (WIMAX), Int. Journal of Engineering Research and Applications www.ijera.com ISSN: 2248-9622,2013, (3), Issue 6, Nov-Dec, pp.1344-1350.

5. Zhu, S., \& Wang, T (2007). Enhanced power efficient sleep mode operation for IEEE 802.16e based WiMAX, in IEEE Mobile WiMAX Symposium, 2007, pp. $43-47$.

6. Xiao, Y. (2005). Energy Saving Mechanism in the IEEE 802.16e wireless MAN, IEEE Communications Letters, 2005, (9), pp. 595-597.

7. Jufeng, X., Zou, S., Ren, B., and Cheng, S. WLC17-6: (2016). An enhanced energy saving mechanism in IEEE 802.16e, in IEEE Global Telecommunications Conference (GLOBECOM'06), 2016 pp.1-5.

8. Yang S (2007). Dynamic Power Saving Mechanism for 3G UMTS Systems Department of Computer Science National Tsing Hua University, Hsinchu, Taiwan, R.O.C.in Mobile Networks and Applications. Pp 1-8.

9. Min-Gon, K. Kang M., and Choi, J. Y. (2008). Remaining Energy-aware power Management mechanism in the 802.16e mac, in 5th IEEE Consumer Communications and Networking Conference (CCNC'08), 2008, pp. 222- 226.

10. Wisdom, D.D., Saidu, I, Tambuwal, A.Y.,Ahmed, M.A., laac, M., Farouk, N (2019). An EfficientSleep-Window- Based Power Saving Scheme in IEEE 802.16e Networks in 15th International Conference in Electronic Computer and Computation (ICECCO), Nile University, Abuja, Nigeria, 10th to 12th December 2019.

11. Jianbing X., Zhanting Y., and QiuYu Z (2008). Traffic Load-Aware Power-saving Mechanism for IEEE 802.16e Sleep Mode, in College of Computer and Communication Lanzhou University of Technology Lanzhou, China. 
12. Eunju H., Kyung J.K., Jung, J. S. and Bong D. C (2007). The Power Saving Mechanism with Periodic Traffic Indications: A New Sleep Mode Scheme in the IEEE 802.16e, Proceedings of the Third Korea Netherlands Conference on Queueing Theory and its Applications to Telecommunication Systems. 2007, pp. 319-334. Saidu, I.,

13. Musa, H., Lawal, M. A., \& Kane, I. L. (2017). Hyper-Erlang Battery-LifeEnergy Scheme in IEEE 802.16e Networks, Covenant Journal of Informatics \& Communication Technology 5(2), pp. 7178.

14. Wisdom, D. D., Tambuwal, A. Y., Mohammed, A., Audu, A., Soroyewun, M. B., and Isaac, S., (2019). A DelayAware Power Saving Scheme (DAPSS) Based on load in Traffic in IEEE 802.16e WiMAX Networks, Institut of Electrical and Electronic Engineers Conference (IEEE), Ahmadu Bello University Zaria, Kaduna State, Nigeria

15. Wisdom, D.D., Ahmed, M. A., Isaac S., Idris H., Farouk N and Hassan J.B (2020). An Enhanced Power SavingScheme Based on load in Traffic in IEEE 802.16e Networks, International Journal of Information Processing and Communication (IJIPC) 2020.

16. Wisdom, D.D., Ahmed, M. A., Isaac S., Farouk N., Magami S., and Elijah Y (2020). Improving Efficiency with a Battery Life Time Aware Power Saving Scheme in IEEE 802.16e Networks, International Journal of Information Processing and Communication (IJIPC) 2020.

17. Chou, L.D., Li, D. C., and Hong, W.Y (2013). Improving energy efficient Communications With a battery lifetime-aware mechanism (BLAPS) in IEEE 802. 16e Wireless networks. Concurrency and Computation: Practice and Experience, (25), 2013, pp. 94-111.

18. Lin, Y-W., and Wang, J-S (2013). An Adaptive QoS Power Saving Scheme for Mobile WiMAX, Wireless Pers Commun, (2013) 69:1435-1462.

19. Saidu, I. Subramaniam S, Jaafar A, Zukarnain Z. A (2015). An efficient battery lifetime aware Power saving (EBLAPS) mechanism in IEEE 802.16e networks. Wireless Personal Communications 2015, (80), pp. 29-49.

20. Zhang $Y$ (2007). Performance Modeling of Energy Management Mechanism in IEEE 802.16e Mobile WiMAX, WCNC 2007 Proceedings. PP. 3207-3211.w 